\title{
Entre individualisation et responsabilités familiales : les mobilités des jeunes de la région de Kayes au Mali
}

Between Individualization and Family Responsibilities: Mobilities of Young Adults Coming from the Region of the Kayes in Mali

Entre individualización y responsabilidades familiares: las movilidades de los jóvenes de la región de Kayes en Mali

\section{Christophe Daum}

\section{(2) OpenEdition \\ Journals}

Édition électronique

URL : https://journals.openedition.org/remi/7009

DOI : $10.4000 /$ remi.7009

ISSN : $1777-5418$

Éditeur

Université de Poitiers

Édition imprimée

Date de publication : 1 décembre 2014

Pagination : 163-180

ISBN : 979-10-90426-23-8

ISSN : 0765-0752

\section{Référence électronique}

Christophe Daum, «Entre individualisation et responsabilités familiales : les mobilités des jeunes de la région de Kayes au Mali », Revue européenne des migrations internationales [En ligne], vol. 30 - n³ et 4 I 2014, mis en ligne le 01 décembre 2017, consulté le 14 avril 2022. URL : http:// journals.openedition.org/remi/7009; DOI : https://doi.org/10.4000/remi.7009 


\section{Entre individualisation et responsabilités familiales : les mobilités des jeunes de la région de Kayes au Mali}

\section{Christophe Daum ${ }^{1}$}

Cet article explore les différentes mobilités des jeunes gens de la région de Kayes au Mali, à partir d'une enquête menée en 2010 et $2011^{2}$. Cette région, et principalement sa partie soninké en rive droite du fleuve Sénégal, est connue pour son émigration vers la France depuis les années 1960. Mais les jeunes générations semblent expérimenter des solutions différentes en termes de rapport à l'emploi et de stratégies d'individuation. En effet, l'ampleur de leurs mobilités vers Bamako, la capitale nationale, d'une part et la situation de " nonemploi " de la quasi-totalité des émigrés de la zone de Kayes avant leur départ d'autre part, constituent deux éléments remarquables qu'il s'est agi d'expliciter plus avant. On le verra, ces mobilités spatiales sont principalement motivées par des stratégies visant à la mobilité sociale.

Pour éclairer les stratégies de ces jeunes gens, les chefs de famille (diatiguỉ) à Bamako de cinquante-trois " maisons d'accueil " des ressortissants des villages concernés ont été rencontrés. Le dénombrement des personnes accueillies a d'abord été réalisé (les critères de ce dénombrement incluant les variables suivantes : genre, âge, motif et durée du séjour à Bamako, village d'origine). Ces maisons accueillaient alors 670 personnes (soit une moyenne par maison de près de treize personnes étrangères à la famille directe du logeur, laquelle peut être elle-même d'une taille conséquente). Dans un second temps, 200 de ces personnes ont été questionnées individuellement. Enfin, une cinquantaine de jeunes gens se sont prêtés à des groupes de discussion pour affiner la compréhension de leurs différentes perspectives. Ces dernières s'organisent, finalement, autour d'une alternative : étudier ou émigrer.

\footnotetext{
1 Sociologue, Maître de conférences, Dysola, Université de Rouen, UFR SHS, Rue Lavoisier, 76821 Mont-Saint-Aignan ; christophe.daum@univ-rouen.fr

2 Il était prévu de continuer ce travail en 2012 pour approfondir un certain nombre de questions apparues au fil du terrain (en particulier, la composition familiale des jeunes gens rencontrés, ou encore les rapports de genre). Les évènements de mars 2012, qui ont trouvé l'auteur de cet article à Bamako, ont évidemment remis en cause ce programme.

3 En Bambara, langue véhiculaire au Mali, le logeur, chef de famille de la maison qui accueille. En découle le terme de diatiguiya, " sens de l'hospitalité ".
} 
L'enquête auprès des diatigui dégage une première typologie des motifs de séjour dans cette ville et dessine quelques repères. Deux groupes, concernant principalement des jeunes gens, attirent plus particulièrement l'attention : ils totalisent plus de la moitié de la catégorie des jeunes et $35 \%$ du total des 670 personnes accueillies dans ces maisons. Il s'agit d'une part des « jeunes en formation " (du secondaire à l'enseignement supérieur en passant par la formation professionnelle), d'autre part des " candidats à l'émigration internationale ". Le premier groupe est relativement mixte (un tiers de jeunes femmes), tandis que le second est très majoritairement masculin.

Tout semble distinguer a priori ces jeunes gens dans les projets de vie qu'ils forment et les stratégies qu'ils dessinent. Les uns affirment vouloir se former pour "vivre et travailler au pays ", tandis que les autres aspirent au contraire à quitter le pays pour travailler en émigration. Ils sont pourtant originaires des mêmes villages et confrontés aux mêmes conditions socio-économiques. Si le statut social est valorisé dans une hiérarchie encore assez rigide dans la région d'origine (structurée principalement autour de deux catégories : les gens d'origine libre et ceux qui descendent d'esclaves), il est relativement secondaire dans le quotidien de Bamako et la diversité des origines régionales. II ne constitue que très marginalement une ressource. Ils sont aussi membres de mêmes classes d'âge ${ }^{4}$, voire appartiennent à une même fratrie dans certains cas. Enfin, ils relèvent tous d'une génération qui, contrairement à celles de leurs aînés (pères, oncles, frères) émigrés entre les années 1970 et 1990, n'a connu comme seule source de sécurisation de l'économie domestique que les revenus transférés par les émigrés. En effet, c'est au tournant des années 1970 que la crise de l'agriculture traditionnelle prend toute son ampleur, les périodes de grandes sécheresses touchant le Sahel et révélant brutalement la faillite des économies d'autosubsistances dans ces régions. Si dans les premiers temps on espère encore que la crise agricole ne sera que passagère, très vite celle-ci $s^{\prime}$ avère définitive. Dès lors, l'émigration vers la France va changer de nature. Sa raison d'être à l'origine était d'obtenir des revenus complémentaires à ceux de l'économie agricole, mais, depuis le milieu des années 1970 en effet, la survie quotidienne des populations demeurées au pays dépend très principalement des salaires de l'émigration : chacun le sait au village (Quiminal, 1991 ;Timéra, 1996 ; Daum, 1998). Quarante ans après, le fait est devenu incontournable : la migration est inscrite de façon structurelle dans les économies domestiques et villageoises.

C'est dans cet univers que ces jeunes gens, issus du milieu rural de Kayes et résidant à Bamako, ont été socialisés depuis leur enfance ; cet élément imprègne de façon permanente les références collectives. Arrivant à l'âge adulte, ils sont impatients de construire leur avenir social et professionnel ; l'émigration constitue une option bien rôdée dans leur univers. Elle fait, en quelque sorte,

4 "Classe d'âge " entendue ici dans une double acception : celle, démographique, qui range ces jeunes dans une même génération ; celle, sociale, qui indique en pays soninké et plus largement en pays musulman, que ces garçons, majoritaires dans le panel ici questionné, ont été circoncisés en même temps. Ils relèvent alors de la même association de jeunes (lemine fedde) et ceci implique une forte solidarité entre ses membres. Les mariages, la construction de la maison, les baptêmes des enfants, la fête nationale et I'achat d'un mouton voir d'un bœuf en commun, etc. réactivent régulièrement ces liens générationnels. 
partie de I'héritage familial, comme d'autres hériteraient de la terre agricole ou d'un savoir-faire professionnel et éventuellement des outils afférents à la fonction. Toute l'expérience de la migration, acquise par leurs aînés, appartient ainsi à leur capital tant culturel que social. Pourtant ce qui diffère entre ces jeunes, c'est le projet qui les amène à séjourner à Bamako : les uns y suivent une formation, les autres sont en attente du départ espéré pour l'Europe. Mais, in fine, ils ont en commun d'arriver à l'âge où la société attend d'eux qu'ils assument leurs responsabilités d'adulte vis-à-vis de leur collectivité et, d'abord, de leur famille. Or, "pour les jeunes citadins, la seule vraie reconnaissance passe par l'accès à l'emploi rémunérateur. C'est la condition de leur entrée dans I'espace public comme individus majeurs ayant un statut social " (Timéra, 2001 : 41).

Leur habitat en ville s'inscrit dans un système complexe, fait à la fois de proximité sociale et d'éloignement spatial. Proximité sociale en effet, car ces villages ont tous dans la capitale plusieurs diatigui chez qui I'hospitalité, pour un soir ou pour plusieurs mois, est assurée. Mais ces jeunes sont néanmoins confrontés aux mêmes difficultés quotidiennes, dépendant d'envois d'argent rarement réguliers de leurs parentés émigrées pour se rendre sur leurs lieux d'étude, prendre leur repas du midi, etc. Éloignement spatial, car la multiplicité de ces logeurs et le fait qu'ils soient établis dans des quartiers éloignés les uns des autres font que deux personnes, par ailleurs amies au village, mais séparées par des trajectoires différentes, éventuellement parallèles, peuvent ignorer qu'elles résident simultanément à Bamako, chez deux logeurs différents. Ainsi, ces maisons, accueillant des ressortissants de villages relativement voisins et entre lesquels les échanges sont nombreux, sont situées dans quinze quartiers dispersés sur l'ensemble de la capitale.

\section{La diatiguiya, une obligation sociale parfois complexe}

Dans les débuts de l'émigration des gens de Kayes vers la France, les voyages étaient essentiellement assurés par bateau et dès lors au départ de Dakar, le transport aérien étant encore très onéreux. En outre, I'enclavement de la région de Kayes vers Bamako rendait la capitale nationale plus difficile d'accès que Dakar, malgré les distances (environ 600 kilomètres pour Bamako contre 900 pour Dakar, mais avec un réseau routier à l'époque plus performant au Sénégal). Samuel (1977), puis Quiminal (1991) décrivent le contexte de ces séjours à Dakar. Très vite, les ressortissants du cercle de Kayes avaient fait construire à Dakar une maison, véritable "foyer pour célibataires " tel qu'ils pouvaient le pratiquer en France, afin d'y loger leurs gens en transit, soit revenant au village pour les congés soit se préparant à partir en France. II s'agissait également de travailler à Dakar pour gagner le prix du transport maritime. Quiminal explique que ce temps passé dans la capitale sénégalaise - jusqu'à un an - permettait en outre de se familiariser avec la ville, cette période offrant une transition en douceur entre le mode de vie rural et le mode de vie urbain.

À Bamako même, où les séjours de ressortissants de Kayes étaient à cette époque généralement de courtes durées, I'hospitalité offerte par les gens de la région établis comme commerçants ou, plus rarement, fonctionnaires dans la 
capitale nationale, permettait de s'y loger le temps de régler ses affaires. Cette hospitalité parfois transmise d'une génération à l'autre est, encore aujourd'hui, accordée en fonction de critères assez souples. L'appartenance au même village, à un même lignage en cas d'appartenance villageoise différente, à un lignage allié à celui du chef de famille, par exemple à la suite d'échanges matrimoniaux, voire le rappel de ce que le grand-père du logeur et celui du visiteur étaient amis, suffisent encore de nos jours pour se faire attribuer une place où dormir et avoir I'assurance de manger lors des repas. Toujours est-il que le proverbe, largement plus bamakois que spécifiquement kayésien, affirme qu'il "vaut mieux ne jamais revenir dans un village (une ville, un quartier éloigné, etc.) que de ne pas loger chez son diatigui du premier séjour ". C'est dire si ce devoir d'hospitalité constitue une obligation sociale forte et implique une nécessaire fidélité.

Dans les années 1970 et 1980, les vols intercontinentaux vont se démocratiser et devenir accessibles à un plus grand nombre. L'étape dakaroise va péricliter et être de plus en plus supplantée par l'étape bamakoise. Mais l'hospitalité des diatiguis va continuer à constituer le principal mode d'accès au logement pour les visiteurs à Bamako. Insensiblement, les diatiguis vont devoir faire face à une augmentation du nombre de personnes accueillies et également à l'amplification de la durée des séjours. Les personnes accueillies indiquent généralement qu'elles versent une somme d'argent en contrepartie de l'hospitalité qui leur est accordée, mais cette compensation n'est pas toujours équivalente ni régulière. Cette hospitalité constitue de nos jours une lourde charge pour les logeurs.

\section{La diversité des motifs de séjours}

La répartition des 670 personnes accueillies se distribue autour de six motifs principaux de séjour.

- 145 jeunes gens résident à Bamako pour y suivre une formation (scolaire, professionnelle, universitaire) et constituent un premier groupe.

- Dans cette même tranche d'âge, 142 " attendent le visa ", dit autrement, ils sont candidats à l'émigration.

- 119 personnes ont fait le déplacement à Bamako pour des raisons familiales : baptêmes, mariages ou décès sont autant de moments sociaux respectés.

- Quatre-vingt-onze ont fait le déplacement pour des raisons de santé, principalement des femmes, on le verra.

- Quatre-vingt-dix-sept personnes sont venues à Bamako pour y régler des affaires, administratives ou commerciales.

- Enfin, soixante-quinze sont à Bamako en transit : il s'agit là d'émigrés (généralement établis en France) de passage à Bamako, ils arrivent d'Europe et se dirigent vers le village ou, inversement, se préparent à retourner en Europe.

Ces différentes catégories de personnes ont en commun de savoir chez qui, lorsqu'elles arrivent à Bamako, elles logeront. Mais la durée des séjours diffère : le migrant en transit ne passera généralement qu'une semaine dans la capitale, que sa destination ultérieure soit le village ou l'Europe. Tandis que le jeune scolarisé sera présent sur la durée de l'année scolaire a minima. Pour les candidats à l'émigration, personne ne sait quand l'argent du passage arrivera. Quant à la personne montée à la capitale pour s'y faire soigner, on suppose qu'elle retournera au village une fois guérie, mais les choses se compliquent parfois. 
Pour nombre des membres de ces groupes néanmoins, les frontières entre ces catégories sont parfois ténues et le basculement de l'une à l'autre suscite bien des interrogations.

Les migrants en transit à Bamako sont parmi les moins nombreux de I'ensemble des personnes accueillies : ceci n'est guère surprenant. En effet, si les nombreux allers-retours des émigrés entre France et Mali représentent une caractéristique constante de cette émigration ${ }^{5}$, le nombre des voyageurs n'est pas extensible, mais dépend des périodes et des opportunités de voyage. Cet effectif peu élevé est indiqué par les logeurs comme étant assez régulier, et ce fait témoigne de la permanence des liens entre les deux pôles principaux de la chaîne migratoire des ressortissants de Kayes, la France et le Mali.

Les femmes sont relativement minoritaires dans cette population, dont elles ne représentent qu'un peu plus du tiers. La séparation des sexes se retrouve toutefois dans les motifs du séjour : ces derniers apparaissent clivés selon le genre.

En effet, près de $60 \%$ des hommes apparaissent tournés vers "l'ailleurs ", que ce soit à travers la formation, les affaires ou le projet d'émigration. Tandis que plus de la moitié des femmes réside dans la capitale pour des raisons d'ordre privé : en vue d'y recevoir des soins ou, en proportion plus importante encore, pour une visite familiale.

On sait que le " séjour à Bamako pour soin " des épouses d'émigrés installés en France peut parfois participer d'une stratégie de pression envers les maris, afin de leur faire accepter le regroupement familial et l'émigration de l'épouse et des enfants éventuellement, vers la France. Azoulay et Quiminal (2002 : 87) notent ainsi que " même lorsqu'elles le souhaitent, le départ des femmes mariées en migration se fait sur décision du mari, après accord du chef de famille responsable du ka (maisonnée regroupant trois ou quatre générations, unité de production agricole et de reproduction) ". Dans le même ordre d'idées, séjourner à Bamako au motif de se soigner permet d'échapper un temps à la " dépendance accrue par rapport à la famille de leur mari " (Quiminal, 1998 : 72). Ces deux invocations - émigrer à leur tour ou échapper à leur situation de belle fille - peuvent bien entendu être liées. Toutefois, que ces séjours soient ou non des prétextes dissimulant une stratégie d'émigration, il importe ici de relever que la légitimation de la présence des femmes à Bamako est principalement rapportée à des affaires d'ordre privé. On mesure l'une des limites de la typologie présentée plus haut : le prétexte médical recouvre parfois des motifs non explicités, renvoyant à une perspective plus dynamique que le simple statut passif de malade qui reçoit des soins.

Le groupe des écoliers, étudiants ou encore jeunes en formation représente la première catégorie par ordre d'importance, avec plus d'une personne sur cinq (près de $22 \%$ de l'ensemble). La pratique consistant à organiser l'accueil en ville d'élèves le temps de leurs études se rencontre dans maints autres pays d'Afrique de l'Ouest, comme le relève Pillon (2003 : 16) : « À partir du collège, la

5 Cette caractéristique est largement éclairée par des recherches précédentes. Voir notamment Samuel (1975), Quiminal (1991) et Daum (1998). 
localisation des établissements scolaires dans les centres urbains contraint les élèves et étudiants originaires des zones rurales, pour ceux dont les familles le peuvent, d'aller dans des internats, le plus souvent de se faire héberger par des familles résidant en ville, ou encore d'avoir son propre logement ". Si I'on trouve également des "enfants confiés " dans la région de Kayes, il faut néanmoins relever les spécificités de la scolarisation des jeunes de cette région à Bamako. En effet, dans le cas présent, c'est rarement chez des parents directs que les élèves et étudiants sont logés, puisqu'au contraire, ils ont intégré le système de la diatiguiya afin d'étudier dans la capitale. Cette adaptation de l'institution, originellement destinée à des accueils ponctuels et qui assume une nouvelle demande sociale - le logement des jeunes scolarisés dans la capitale - témoigne de sa vitalité.

Les jeunes femmes représentent par ailleurs un peu plus du quart du groupe des élèves et étudiants. Cela est loin d'être marginal, rapporté au taux de scolarisation des filles au Mali. En 2009 en effet, la statistique nationale relève que 64,7 \% des filles de la région de Kayes sont scolarisées au premier cycle de l'école fondamentale (la moyenne nationale est alors de $72 \%$ ), et $25 \%$ des filles au second cycle (près de $42 \%$ au niveau national). Ces proportions, qui peuvent en première lecture apparaître très faibles, sont en réalité à inscrire dans une progression constante et notable pour la région de Kayes ces dix dernières années.

Le groupe des candidats à l'émigration est le second par ordre d'importance avec plus de $21 \%$ de l'effectif total des personnes accueillies. II est composé à plus de $90 \%$ par des jeunes hommes, avec une moyenne d'âge de vingthuit ans. II compte d'autre part pour presque $30 \%$ du total des hommes, chez lesquels il représente le premier motif de séjour à Bamako. Ces jeunes gens se caractérisent par l'absence d'occupation régulière en ville : seuls sept d'entre eux indiquent avoir une occupation de type professionnel (petit commerce, tailleur) et vingt-six déclarent suivre un apprentissage à Bamako. Les quatre cinquièmes déclarent ne rien faire d'autre que d'attendre. Tous, finalement, donnent la priorité à l'attente du visa : " je cherche la route ", ou encore "j'attends le visa (pour la France) ", sont des formulations qui reviennent régulièrement dans leurs propos. En réalité, pour reprendre une formulation de Sayad (1975: 54) : " [...] les uns comme les autres [...], dans la mesure où c'est toute leur pratique quotidienne qui est déterminée par le projet de l'émigration, ne vivent au village [et de même à Bamako dans le cas présent] que "provisoirement", comme "s'ils n'avaient à y passer que des vacances" ". Leur situation à Bamako, vécue comme une transition entre le village et la France, légitime à leurs yeux un réel désœuvrement, tout autant sans doute qu'elle est vécue comme un pis-aller par la famille qui assume financièrement la situation.

Ces candidats à l'émigration sont largement majoritaires dans les tranches d'âge des vingt à trente-neuf ans, avec $94,5 \%$, et près de la moitié de leur groupe se situe dans la tranche des vingt-cinq à vingt-neuf ans. Émigrer demeure bien une affaire de jeunes gens, élément constaté par de nombreuses recherches sur les mobilités.

Enfin, les visites familiales se rencontrent plus fréquemment chez les plus de trente ans, ainsi que le séjour pour soins. C'est également dans ce groupe d'âge que les migrants se situent. 
Finalement, les raisons du séjour recouvrent pour partie le cycle de vie, avec la période de la construction du projet individuel, mais également, à l'âge adulte, les échéances plus marquées des sociabilités familiales ou encore du recours aux structures de santé.

Le groupe des élèves et étudiants retient plus particulièrement l'attention. En effet, il se caractérise par la constance de sa présence. Les plus anciennement installés à Bamako ont pu y faire le second cycle et s'inscrire ensuite à l'université. Ces derniers totalisent parfois de cinq à sept ans de séjour.

Tandis que près de la moitié de ceux qui totalisent moins d'un an de séjour (le quart environ du total des personnes interrogées) envisage d'émigrer, soit plus des deux tiers des candidats à l'émigration.

Différentes possibilités peuvent expliquer cette polarisation. La première est que le séjour à Bamako peut constituer une étape relativement courte d'un projet migratoire programmé à l'avance, organisé généralement par un frère émigré lui-même. Ainsi, plusieurs de ces jeunes indiquent être sur le départ. La deuxième possibilité est que des jeunes, lassés d'attendre le visa, retournent provisoirement au village : ils ne sont plus que dix à avoir séjourné dans cette attente entre un et deux ans, et neuf ont séjourné entre deux et trois ans, à la suite de quoi les effectifs s'effondrent. À moins qu'ils aient changé de catégorie en s'inscrivant dans un projet de formation ou en se lançant dans une petite affaire de type commerce, afin d'occuper leur attente à Bamako. En effet, leurs pères, oncles ou grands frères émigrés, susceptibles de leur payer le visa, peuvent laisser trainer leur réponse et miser sur le découragement de leurs cadets. Ceci constitue de leur part une forme de résistance face aux pressions de ces jeunes : l'investissement financier est important et suppose, dans le contexte français actuel, une prise de risque certaine. Cet élément explique sans doute la fréquence des séjours à Bamako des candidats à l'émigration : soixante d'entre eux - sur soixante-treize - indiquent y avoir effectué au moins un séjour antérieur.

\section{Étudier ou émigrer?}

Cette alternative est relativement récente dans la région de Kayes. En effet, la zone où se situent les villages d'origine est passée de sept à 450 écoles en vingt-cinq ou trente ans. La statistique du ministère de l'Éducation nationale indique que, entre 2007 et 2010 , la population scolarisée dans le premier cycle a augmenté de $18 \%$ (plus $19 \%$ pour les garçons et 16,30\% pour les filles). La même statistique indique que les taux bruts de scolarisation pour la région de Kayes, s'ils sont généralement inférieurs à ceux de l'ensemble du pays, se tiennent dans une fourchette honorable vis-à-vis de la désaffection scolaire auparavant constatée dans cette région qui s'est longtemps refusé à scolariser ses jeunes générations (Daum, 1998).

Depuis 1991 au Mali, année du renversement d'une dictature de parti unique instaurée par un putsch militaire en 1968 et de la démocratisation suivie de l'instauration de la III ème république, ce pays s'est confronté avec volontarisme au défi de la scolarisation de sa jeunesse, défi symbolisé par la formule "pas de village sans école ". Cette volonté politique a conforté les associations villa- 
geoises des émigrés de France, qui avaient, dès le milieu des années 1980, milité en faveur de l'importance de la scolarisation des enfants et financé la construction d'écoles dans les villages d'origine. On sait par ailleurs (Cissé et Daum, 2009) que l'utilisation au village de l'argent envoyé par les émigrés aux chefs de famille porte sur trois postes prioritaires de dépenses : la scolarisation des enfants apparaît en seconde position, après I'alimentation familiale et avant la santé.

De façon générale, les jeunes rencontrés à Bamako sont en situation de mobilité. Il s'agit d'abord de mobilité spatiale : de la campagne vers la ville. Mais celle-ci est destinée à leur permettre un tout autre type de mobilité, sociale celle-là. Que leur objectif soit l'émigration ou la formation, leurs stratégies visent à répondre à une exigence : accéder au statut d'adulte reconnu comme tel auprès de la famille. Or, si " les jeunes sont pris en charge pour la nourriture et I'hébergement, ils se trouvent privés de la gestion d'un espace économique individuel et privé ". Mais "I'affirmation individuelle se réalise à la fois dans l'acte d'avoir et de donner. La souffrance des jeunes est autant de ne pas avoir que de ne pas pouvoir rendre aux aînés " (Timéra, 2001 : 43). Ces jeunes sont conscients des réalités complexes auxquelles ils sont confrontés. Leurs aspirations impliquent en effet, dans tous les cas, un glissement statutaire fort : du paysan astreint à un revenu annualisé par le cycle agricole - combien fragilisé et aléatoire dans les zones sahéliennes - au salarié dont le revenu est mensualisé et en principe sécurisé. Ce lent processus de déclassement - reclassement a été analysé dans un autre contexte historique par Bourdieu et Sayad (1964), concernant les émigrés algériens originaires de Kabylie passant insensiblement du statut de paysan à celui d'ouvrier. L'émigration ou l'accès à un emploi dans le secteur moderne du Mali représentent ainsi les deux facettes d'une même logique, dans un contexte qui a fortement changé depuis une vingtaine d'années en matière de scolarisation.

Comment se positionnent ces jeunes, confrontés à ces différentes options ? Quelles sont leurs ressources ? Et enfin, comment leurs projets sont-ils susceptibles de se transformer au fil de l'expérience ? Ainsi, l'alternative "émigrer ou étudier " paraît être en débat au sein même des familles et entre les jeunes. La frontière entre les différents groupes de jeunes gens, étudiant, en apprentissage ou à I'école, ou attendant le visa pour la France sans rien faire de ses journées, est relativement ténue, avec des basculements possibles de l'une à l'autre de ces deux catégories, dans les deux sens. Selon leur projet et selon leurs ressources ou encore leur statut, ces jeunes ne vivent pas de la même façon la situation dans l'espace urbain de Bamako et ils investissent différemment I'avenir. Tous ont néanmoins comme référence l'émigration en France, de préférence à d'autres pays de destination : soit qu'elle constitue leur objectif, soit que les émigrés de leurs familles qui y sont établis soutiennent financièrement leur cursus de formation.

\section{Les candidats à l'émigration}

De même âge et parfois de même fratrie que les élèves et étudiants, ceux qui attendent leur visa sont dans une logique de reproduction de ce qu'ont fait leurs aînés, " il n'y a que par l'émigration que je vais pouvoir faire quelque chose de ma vie et assumer la famille ". Ils tiennent un discours individualisé sur le 
parcours qu'ils vont suivre, désirent partir à l'aventure et veulent prendre leurs responsabilités à leur tour. Leurs carrières scolaires sont d'évidence en échec : ils ont très souvent abandonné l'école fondamentale, parfois après plusieurs redoublements successifs et ayant atteint la limite d'âge de scolarisation. Ainsi, plus des deux tiers des membres de ce groupe ne déclarent aucun niveau de formation initiale.

Ces jeunes expliquent leur vie de tous les jours au village et l'absence de perspectives: " si tu ne vas pas à l'école et si ce n'est pas l'hivernage (la saison des cultures), il n'y a rien à faire ". Mais ils ajoutent que, sans formation professionnelle ils n'ont pas de possibilités d'emploi et ne peuvent dès lors pas " assumer la famille ": " nos aînés sont partis avant, c'est à notre tour d'aller chercher de l'argent ailleurs, de partir à l'aventure afin de pouvoir assumer nos responsabilités ". Pourtant, les activités ne manquent pas dans l'économie domestique, mais celles-ci n'offrent pas ou très peu d'opportunités individuelles tant les budgets sont resserrés et les équilibres fragiles. Tout ce qui permet un gain en numéraire (ramassage de bois de chauffe, petit emploi occasionnel de réparateur de vélo ou moto, petit commerce de détail, etc.) est mesuré au plus près, mais va généralement alimenter le grenier familial - pris ici au sens le plus large englobant l'ensemble des ressources de la maisonnée - afin de sécuriser du mieux possible la vie quotidienne de celle-ci. Dans cette conjoncture, I'autonomisation des jeunes n'est rendue possible que par une activité extérieure à la famille, quand bien même ils ont l'assurance que leurs besoins primaires, alimentation et santé, y sont satisfaits.

Finalement, le quotidien de ces jeunes est bien assuré ; mais une question, lancinante, demeure, celle de savoir comment la relève intergénérationnelle sera assurée dans ces économies fortement organisées autour des revenus issus de l'émigration. La formulation fréquemment entendue, "il nous revient de prendre en charge nos responsabilités face à la famille " prend alors tout son sens : I'une des façons d'assumer cette contrainte est bien d'émigrer à leur tour.

II n'en demeure pas moins que l'attente à Bamako se prolonge souvent plus longtemps que leur espérance initiale le laissait espérer : le coût du visa a connu une véritable inflation. Les parents émigrés en France, auxquels il revient de financer le passage de leurs cadets, hésitent donc devant un investissement dont ils connaissent tant les coûts que les risques et, partant, la possibilité que les dépenses soient perdues à la suite d'une expulsion de France au terme de l'aventure. Les émigrés, confrontés aux pressions de leurs cadets, peuvent y répondre indirectement et de façon négative, en laissant trainer les décisions et patienter leurs jeunes à Bamako. Tant il est vrai, comme le relevait déjà Quiminal en 1991, que ne partent pas en émigration ceux qui le souhaitent, mais ceux qui sont choisis par la famille, souvent après une période de test. II s'agit d'avoir l'assurance que le futur émigré réunit les garanties nécessaires à la réussite de l'émigration. Fidélité à la famille et courage devant le travail, esprit d'économie et, plus largement, respect des valeurs sociales sont parmi ces caractéristiques et représentent autant de qualités morales permettant d'assurer que l'émigré 
honorera sa dette envers le groupe familial ${ }^{6}$. Le phénomène est très similaire à celui que Sayad (1977: 62) décrit concernant la première phase du cycle migratoire algérien ("le premier âge de l'immigration ") : "Au service du monde paysan, subordonnée à l'activité agricole dont elle était un appoint, l'émigration sélectionnait ses agents selon les principes de l'habitus paysan ".

Nous sommes dans une logique de sélection et de contrôle des départs par les membres des sociétés d'origine, sélection qui s'opère principalement au sein de la famille : c'est elle qui attend une aide des émigrés dans le même temps qu'elle cautionne les départs. Dans un contexte durci par les politiques migratoires européennes, la sélection s'avère difficile.

\section{Les élèves et étudiants}

Le discours assez dominant au sein de ce groupe s'inscrit dans un registre complexe, mobilisant au moins trois niveaux. Tout d'abord, ces jeunes, et particulièrement les étudiants, portent un regard critique sur leur région d'origine, dont ils aspirent au développement. Dans un second temps, I'appartenance collective dont ils se réclament, autour de l'identité soninké, est discutée. Finalement, tous font part de leurs aspirations professionnelles et de leurs ambitions d'avenir. Ces trois registres articulent la conscience de leurs handicaps, mais également de leurs ambitions professionnelles. Ils expriment leur volonté de s'inscrire dans le monde contemporain sans renier pour autant leurs aînés ni leur héritage tout comme ils dévoilent l'inventaire de leurs ressources.

En ce qui concerne leur région d'origine, la région de Kayes, ils s'affirment, avec une fierté certaine, comme "les premiers intellectuels de leurs villages". Et, de fait, leur génération est la première à compter des étudiants en premier ou second cycle. Le regard qu'ils portent sur leurs aînés, pères, oncles ou frères, est en double teinte. Ces derniers ont émigré dans de mauvaises conditions : généralement analphabètes ils ont été employés aux postes de travail les plus durs et les moins bien payés. D'un côté le respect dû à leurs aînés est très présent ; de l'autre ils sont animés d'une compassion certaine vis-à-vis de ce qu'ils considèrent comme une vie sacrifiée. Ils savent pourtant que ce sont précisément les caractéristiques de leurs aînés sur le marché du travail, leur absence de qualification, qui les ont rendus disponibles et les ont fait recruter par les entreprises françaises.

Leur cursus scolaire, voire universitaire, est en rupture avec tout cet héritage, tant régional que familial. Il suppose un certain volontarisme : en effet, les élèves et étudiants expliquent que leur capital social est de faible envergure à Bamako, en tout cas à l'échelle de leurs relations de proximité. Dès lors, ils savent n'avoir que très peu d'appuis dans la capitale, parmi les cadres supérieurs et autres professions valorisées, à même de les aider à trouver un emploi correspondant à leurs diplômes ou à leurs ambitions. "Même si tu es le meilleur dans un concours, si tu n'es pas pistonné tu ne seras pas classé ", affirment-ils. Mais, en

6 Quiminal (1991) parle à ce sujet de la double dette : celle, financière, qui oblige à rembourser le prix du passage en France auprès de ceux des membres de la famille qui ont fait I'avance ; celle, morale et symbolique, qui correspond à I'absence sur les champs du chef de famille, à la ponction de la force de travail que l'émigration représente pour l'économie domestique. 
cela, ils rejoignent l'opinion générale de la jeunesse malienne, et notamment des jeunes diplômés, confrontés à la pénurie des emplois publics depuis les plans d'ajustement structurel. Autre argument, ils indiquent que "la région de Kayes est oubliée, isolée, on n'a pas de piston et alors il nous faut nous battre". Parmi leurs ressources dans cet itinéraire d'intégration au monde du travail, à laquelle ils aspirent, l'origine régionale est mise en avant : puisqu'ils n'ont pas les réseaux sociaux à même de les parrainer pour leur entrée dans la vie active, puisque leur région d'origine est isolée, ils retournent en quelque sorte ce stigmate et travaillent à la promotion de leur région d'origine. Il s'agit sans doute pour eux de faire un atout de cette situation de minoritaires au sein de l'espace national.

Le second niveau de discours, complémentaire de l'appartenance régionale, porte sur la tradition soninké dont ils affirment la nécessité de son maintien et de son renforcement. Un point de repère récent et important dans cette affirmation identitaire est le Festival international initié par l'Association pour la promotion de la langue et de la culture soninké (APS) en février 2011 dans la ville de Kayes ${ }^{7}$. L'Association des élèves et étudiants soninké de Bamako, créée en 2009, était représentée et deux cars avaient été affrétés par les organisateurs de ce festival pour permettre aux résidents de Bamako de faire le déplacement. Sur la cinquantaine de jeunes rencontrés, seuls deux d'entre eux ont été du voyage : un étudiant en géographie ainsi qu'une jeune fille, étudiante en médecine et membre du bureau de l'Association des élèves et étudiants soninké de Bamako, secrétaire aux relations féminines. Néanmoins, tous avaient été informés de l'initiative, qu'ils considèrent comme un événement important et légitimant leur référence à l'identité soninké. Leur discours apparaît d'abord très idéologique ; ils insistent en particulier sur l'importance des valeurs traditionnelles de leur société d'origine, sur la nécessité de les préserver. Mais, questionnés sur le sens de ces valeurs et au fil des échanges, les jeunes finissent par porter des critiques assez précises sur leur société, mettant en cause les modalités des échanges matrimoniaux, la place des femmes et des cadets, les structures hiérarchiques.

Ces jeunes discutent d'abord des " mariages arrangés par les familles ", qui réactualisent régulièrement des alliances interlignagères souvent anciennes dans leur société d'origine : "dans la tradition il y a un certain nombre de choses qui ne collent pas avec le monde moderne. Chez nous, il y a un problème social. Il y a aussi le problème des mariages forcés, pour nous cela ne sera pas possible, on veut pouvoir choisir librement " disent des garçons. L'une des jeunes filles explique ainsi, devant ses amies, mais également devant quelques garçons, ses mésaventures lorsque ses parents l'ont envoyée en vacances au village après son baccalauréat, son oncle paternel désirant lui " présenter son fiancé ". Affirmant vouloir finir ses études, cette proposition l'a fait quitter dès le lendemain le village pour Bamako. Cette interlocutrice se positionne finalement dans une stratégie identique à celles de jeunes femmes de Bamako rencontrées par Doquet dans sa recherche sur leurs stratégies d'autonomisation ${ }^{8}$. Doquet (2011) indique en effet combien les stratégies matrimoniales des parents ne sont pas remises en cause ouvertement, mais que les jeunes femmes contournent le

7 Les participants venaient de différents pays, outre le Mali : Sénégal, Mauritanie, Gambie. Les émigrés de France étaient fortement représentés.

8 Communication orale d'Anne Doquet, juin 2011, Bamako. 
respect de la norme sociale pour s'affirmer. Quiminal (1997) constate un même type de fonctionnement social de la part de jeunes femmes africaines dont les familles ont émigré en France et qui, sans s'opposer frontalement aux projets parentaux en vue d'un mariage homogame et arrangé entre les familles argumentent de leur projet professionnel pour temporiser : "l'assignation à une scolarité plus longue arme les jeunes filles et les met en position de force dans la négociation avec les parents ".

Finalement, ces jeunes femmes rencontrées à Bamako tendent à s'aligner sur ce qui se joue dans la capitale, concernant les rapports de genre.

Dans cette même logique de remise en cause des modalités matrimoniales qui dominent leur région, certains jeunes hommes portent un discours critique sur la polygamie de leurs pères, dont ils expliquent qu'elle est source de conflits entre les épouses, mais également source de problèmes et de jalousies entre les enfants des coépouses, certains pouvant être privilégiés au détriment des autres. L'autre argument invoqué porte sur l'accès au travail, au sens moderne de ce terme, des femmes : " une femme de chez nous peut devenir aujourd'hui infirmière, sage-femme, institutrice. Mais si son époux a plusieurs femmes, alors il y aura des conflits : celle-là, qui travaille, gagnera son salaire, elle ne voudra pas prendre son tour dans les activités de la maison de la même façon que ses coépouses qui ne travaillent pas en dehors de la maison, cela créera des disputes ". Un autre argument, tenu par des garçons, interroge les rapports de genre dans la société soninké. S'il n'y a pas directement remise en cause de la répartition sexuée des emplois, les exemples de métiers invoqués sont en effet classés parmi les emplois dits "féminins " y compris au Mali, il est néanmoins question que les femmes ne se cantonnent plus à leur rôle de femme au foyer dépendante de leur époux, mais accèdent à des emplois supposant un certain niveau de formation professionnelle. Cette accession à la formation, revendiquée comme légitime par les étudiants et étudiantes rencontrées, entraîne aussi comme conséquence la possibilité du recul de l'âge au mariage des femmes.

Autre élément de ce questionnement de la tradition soninké, ils évoquent la hiérarchie sociale : "Moi par exemple je suis soninké, mais mon diamou (patronyme) est 100 \% malinké. C'est I'histoire qui a voulu que mes ancêtres deviennent soninké, mais du coup ma famille est secondaire dans le village ". Dans le cas présent, le jeune homme évoque directement le statut des descendants d'esclaves (kome) dans la hiérarchie soninké. " Ils sont restés des ressortissants de seconde zone, méprisés des hommes libres ", écrivaient Pollet et Winter (1971 : 255) à propos des descendants d'esclaves en milieu soninké. Quarante ans après cette observation, la situation s'est considérablement assouplie, mais les positions statutaires $n^{\prime}$ appellent pas discussion au village : chacun connait sa place dans la hiérarchie, qui opère un clivage fort entre les gens d'origine libre et ceux d'origine servile avant de segmenter ces deux groupes en plusieurs sous-groupes statutairement inégaux. À Bamako, ces positions statutaires sont formellement respectées. Néanmoins, dans la mesure où la vie de ces jeunes est surtout régie par les études, ils ont rencontré des jeunes de leur âge originaires de toutes les régions du Mali, ils ont accédé à une connaissance nouvelle grâce à l'école ou via l'accès à Internet dans les cybercafés. D'autres statuts sont alors valorisés que ceux qui régissent leur société d'origine : étudiant plus ou moins avancé, jeune écolier ou lycéen, apprenti ou sans-emploi, autant de positions 
qui prennent toute leur importance. Le droit à la parole sera alors fonction de l'accès au savoir moderne et les étudiants de ces groupes, indépendamment de leurs origines statutaires - libres ou non libres -, occuperont alors le devant de la scène. Dans le cas de ces élèves et étudiants, le fait de vivre à Bamako, sortis de l'environnement du village et intégrés dans un univers social - depuis la vie du quartier de résidence à celle du campus de l'université de Bamako - brassant toutes les régions du Mali, contribue certainement à cette conscience qui amène à relativiser l'ordre social établi dans les villages d'origine, introduisant la reconnaissance de la compétence indépendamment du rang assigné par la naissance.

Ces élèves et étudiants sont nombreux à articuler l'intérêt collectif et leur promotion individuelle : " Je veux être un grand avocat pour défendre les pauvres ", dit l'un en maîtrise de droit, tandis que son condisciple déclare vouloir être magistrat pour juger les corrompus. Deux autres, en maîtrise de géographie, veulent devenir agent de développement local. L'un d'eux indique également qu'il souhaiterait faire une thèse et devenir chercheur. Un autre garçon, ainsi qu'une jeune fille, tous deux en formation de métiers de la santé, disent vouloir continuer " jusqu'à être médecin, si j'arrive ". Un élève en dessin du bâtiment exprime son souhait de devenir architecte. Autre exemple, un élève en électromécanique parle de la maintenance du générateur électrique de son village pour expliquer qu'il y aura de l'emploi pour lui au village quand il aura son diplôme. C'est d'ailleurs le seul qui fasse état de ses relations : son frère aîné est technicien sur ce générateur, explique-t-il. Un élève en charpenterie métallique explique également qu'il aura du travail dans son village, avec les nombreuses constructions de maisons et les besoins en portes et fenêtres. Leur projet est donc d'accéder à des emplois utiles pour leur pays et leur commune, ce qui constitue la principale motivation à leur formation professionnelle.

Parmi leurs handicaps, ces jeunes sont relativement isolés et très peu organisés à Bamako. Au village, ils sont membres de l'association de la classe d'âge et celle-ci y joue un rôle fonctionnel. À Bamako, deux ou trois classes d'âge sont représentées dans les groupes de discussion, mais cette ressource sociale ne leur sert strictement à rien. De fait, ils sont dispersés d'un bout à l'autre de la ville et ont peu de moyens pour se déplacer, si ce n'est pour aller à la faculté ou l'école : quelle que soit leur situation, ils dépendent de leur grand frère ou père et des envois d'argent de ces derniers afin de vivre à Bamako et $y$ mener leurs études. Autre élément au sujet de leurs difficultés à Bamako, le fait de devoir se soumettre à l'autorité de leur diatigui. Les contraintes sociales ainsi que les conditions de vie chez les logeurs sont dès lors présentées comme peu favorables aux rigueurs du travail scolaire et de la préparation des examens.

\section{Remarques conclusives}

Dans le contexte de ces mobilités ici étudiées, les situations apparaissent fortement dynamiques et évolutives. Elles autorisent quelques remarques portant d'abord sur l'articulation entre les différents types de mobilités, sur les changements sociaux et culturels de la société d'origine ensuite, sur le devenir des dynamiques, notamment scolaires. 
II apparaît d'abord que ces deux types de mobilités, internes au pays et internationales, sont fortement imbriqués. Le séjour à Bamako apparaît comme un moyen utilisé par les jeunes gens de la région de Kayes pour s'inscrire dans la construction de leur avenir. C'est bien pour accéder à un emploi que ces jeunes résident, de façon plus ou moins longue, à Bamako. Avec cet emploi, qu'il soit situé dans le secteur moderne au Mali ou en migration, c'est bien du salaire correspondant dont il s'agit : leur projet est de participer de façon active à la vie de la famille. Les espoirs qu'ils portent, soit sur leur projet d'émigration soit sur celui de leur formation, apparaissent comme relevant de cette même préoccupation, celle d'accéder au statut d'adulte à même d'assumer une place dans la société. Les alternances entre les deux options, émigrer ou étudier et, parfois, une relative ambivalence dans les motivations, sont dès lors relativement logiques. II s'agit de profiter des opportunités qui se présentent afin de multiplier les chances de réussites. Les deux options, "émigrer ou étudier ", apparaissent alors comme deux facettes d'une même stratégie, facettes optionnellement privilégiées tant en fonction des moyens et des ressources que des possibilités qui s'offrent aux intéressés. Et parfois la faiblesse des ressources ne rend possible que la première option.

Néanmoins, les élèves et étudiants paraissent se faire les porte-paroles et éventuellement les acteurs d'une rediscussion des valeurs de la société d'origine. Certaines remises en cause portent sur des éléments importants : les pratiques matrimoniales, la place des femmes dans la société et les relations de genre, ou encore les relations hiérarchiques ne constituent en rien de petites questions : à y regarder de plus près, ces critiques ne mettent pas moins en cause que certains des piliers de l'ordre établi prévalant dans leur société d'origine!

Sans doute ces remises en cause sont-elles embryonnaires et susceptibles de ne pas avoir un grand avenir. Les pesanteurs sociales incitent en effet les individus, éventuellement en rupture au départ, à réintégrer les habitudes collectives dès lors qu'ils sont réinscrits dans le contexte villageois. II n'est pas indifférent de ce point de vue, d'une part que ces remises en cause soient portées par des jeunes, d'autre part que les discussions aient lieu dans la capitale nationale: autrement dit, loin du regard familial et villageois qui jauge et signale rapidement tout comportement qui ne serait pas conforme à la règle sociale.

Pour autant, il n'est pas indifférent que ces points de vue s'expriment dans des collectifs et non dans des situations d'entretien individuel et en tête-à-tête : le discours est donc tenu en public. D'autre part, ces discours sont formulés par des jeunes qui ne sont pas nécessairement connectés entre eux dans la vie de tous les jours à Bamako. Enfin, des jeunes femmes qui revendiquent le libre choix de leur futur conjoint et le droit aux études sont approuvées par les garçons présents lors du groupe de discussion tenu avec elles. Ces différents éléments indiquent que ces discours rencontrent une certaine légitimité, au moins au sein des groupes de pairs, une fraction significative de la jeunesse de leur région d'origine.

$C^{\prime}$ est finalement une société en profonde mutation qui se donne à voir autour de l'étude des mobilités. En effet, les sociétés villageoises de la région de Kayes ont fait reposer leur survie économique et sociale sur l'émigration, depuis le tournant des années 1970. Certes la sortie de cette orientation est loin d'être 
en vue, en témoignent tous ces émigrés qui travaillent en France et envoient une fraction de leurs salaires au village, en témoignent également le nombre important des candidats à l'émigration rencontrés dans les maisons de passage. Mais il n'en demeure pas moins qu'une catégorie encore émergente est en train de monter en puissance, celle des élèves et étudiants. Il est légitime de s'interroger sur la viabilité des stratégies de scolarisation : ces futurs diplômés vont-ils rejoindre la cohorte des jeunes diplômés sans-emploi ? À tout le moins, leur insertion professionnelle n'est-elle pas donnée.

Alors, leurs aînés qui ont soutenu cette stratégie, parmi d'autres stratégies de mobilités visant à assurer l'avenir de la société d'origine, vont-ils persévérer ou abandonner l'entreprise?

Au cœur de ces mobilités, c'est sans doute le projet d'inscrire la société d'origine dans une certaine forme de modernité qui se dessine. En ce sens que les mobilités - versus émigration et survie quotidienne - permettent de maintenir et préserver la société d'origine, mais également que - versus études et prise d'option sur un autre avenir -, elles ouvrent d'autres potentialités. Il s'agit finalement de trouver les transitions entre le statu quo de l'ordre établi et de nouvelles formes sociales. On I'a vu, l'ordre établi organise et valide le lien social : la capacité d'adaptation de la diatiguiya, qui a su intégrer de nouvelles demandes sociales - celle de la prise en charge des élèves et étudiants par exemple - en est l'un des marqueurs. Mais, si l'ordre établi gère le quotidien, il ne permet pas d'anticiper les transformations sociales et peine à les accompagner. À la suite du groupe des émigrés, dont il a été rappelé dans cet article combien il avait été actif depuis plusieurs décennies dans la transformation des conditions de vie locales, mais aussi pour l'appropriation de nouvelles institutions dans la société d'origine - la scolarisation dont il a été question ici en est l'un des points forts -, il est sans doute permis de se demander si le groupe des scolarisés - nouveau pour la région de Kayes, rappelons-le - n'est pas en passe de devenir le futur groupe des novateurs ? Balandier (1971:207) indique déjà que " les rapports estimés naturels entre générations antécédentes et générations suivantes ont été bouleversés. Les premières ont perdu une large part de leur capacité d'information et de formation, de leur autorité. Elles sont marquées par le passé et effectuent leurs évaluations en termes de continuité, alors que leurs descendants se situent dans le temps des changements incessants et expriment leurs évaluations en termes d'à-venir ". Tant il est vrai, au Mali comme ailleurs, que la jeunesse, à laquelle appartient l'avenir tandis que les aînés tardent à lui laisser la place, est porteuse de l'innovation sociale. Tant il est vrai également que " c'est dans ces compromis qu'il sait passer avec l'ordre social, au besoin lui imposer, que l'individu s'affirme comme tel, à la fois libre et inévitablement solidaire " (Bardem, $1993:$ 393). 


\section{Références bibliographiques}

Azoulay Muriel et Quiminal Catherine (2002) Reconstruction des rapports de genre en situation migratoire (Femmes "réveillées ", hommes menacés en milieu soninké), Villes-Ecoles-Intégration Enjeux, 128, pp. 87-102.

Balandier Georges (1971) Réflexions sur une anthropologie de la modernité, Cahiers internationaux de sociologie, 51, pp. 197-211.

Bardem Isabelle (1993) L'émancipation des jeunes : un facteur négligé des migrations interafricaines, Cahier des Sciences Humaines, 29 (Z-3), pp. 375-393.

Bourdieu Pierre et Sayad Abdelmaleck (1964) Le déracinement (la crise de l'économie traditionnelle en Algérie), Paris, Éditions de Minuit, 220 p.

Cissé Pierre et Daum Christophe (2009) Migrations internationales maliennes, recomposition des territoires migratoires et impacts sur les sociétés d'origine, in Migrations internationales, recompositions territoriales et développement, Paris, MAE/IRD, pp. 191-198.

Daum Christophe (2009) Le travail de la terre et le salaire du chantier, à propos des logiques migratoires en région de Kayes au Mali, in Françoise Dureau et Marie-Antoinette Hily Éds., Les mondes de la mobilité : exploration d'un paradigme, Rennes, Presses Universitaires de Rennes, pp. 39-66.

Daum Christophe (1998) Les associations de Maliens en France (migrations, développement et citoyenneté), Paris, Karthala, 253 p.

Dougnon Isaïe (2007) Travail de blanc, travail de noir (la migration des paysans dogon vers I'office du Niger et au Ghana, 1910-1980), Paris, Karthala/Sephis, $279 \mathrm{p}$.

Doumbia Tamba (2002) Les relations à plaisanterie dans les sociétés mandingues, Recherches Africaines, Annales de la Faculté des lettres, langues, arts et sciences humaines de Bamako, janvier-juin, pp. 28-42.

Marie Alain (Éd.) (1997) L'Afrique des individus, Paris, Karthala, 436 p.

Pilon Marc (2003) Confiage et scolarisation en Afrique de I'Ouest : un état des connaissances, in Preparation of the 2003 EFA monitoring report of UNESCO, 32 p. [en ligne]. URL : http://portal.unesco.org/education

Pollet Eric et Winter Grace (1971) La société Soninké (Dyahunu, Mali), Bruxelles, Éditions de I'Université de Bruxelles, 566 p.

Quiminal Catherine (1998) Comment peut-on être africaines en France ?, Journal des anthropologues, 72-73, pp. 49-61.

Quiminal Catherine (1997) Les jeunes filles d'origine africaine en France : Parcours scolaires, accès au travail et destin social, Migrations Études, 78, pp. 1-5.

Quiminal Catherine (1991) Gens d'ici, gens d'ailleurs, migrations Soninké et transformations villageoise, Paris, Christian Bourgois, 223 p.

Samuel Michel (1977) Le prolétariat Africain noir en France, Paris, Maspéro, $262 \mathrm{p}$.

Sayad Abdelmalek (1977) Les trois " âges " de l'émigration algérienne en France, Actes de la recherche en sciences sociales, 15 (15), pp. 59-79. 
Sayad Abdelmalek (1975) Elghorba : le mécanisme de reproduction de l'émigration, Actes de la recherche en sciences sociales, 1 (2), pp. 50-66.

Timéra Mahamet (2001) Les migrations des jeunes Sahéliens : affirmation de soi et émancipation, Autrepart, 18, pp. 37-49.

Timéra Mahamet (1996) Les Soninké en France, d'une histoire à I'autre, Paris, Karthala, 244 p. 


\section{Christophe Daum}

\section{... Entre individualisation et responsabilités familiales : les mobilités des jeunes de la région de Kayes au Mali}

Cet article porte sur les jeunes des villages de la région de Kayes, au Mali, confrontés à la nécessité de prendre leurs responsabilités d'adultes au sein de leur groupe familial et, plus largement, de leur collectivité. Or, la crise de l'agriculture au Sahel fait que celle-ci ne suffit plus aux besoins alimentaires et ne représente plus un métier. Si leurs aînés ont passé ce cap en émigrant vers la France, les nouvelles générations sont face à une alternative, celle d'émigrer à leur tour ou d'étudier afin de se professionnaliser au Mali. Mais cette dernière éventualité demeure fragile. La frontière entre les deux groupes, candidats à l'émigration ou élèves et étudiants, apparaît alors ténue. Quoi qu'il en soit, le projet de mobilité sociale auquel aspire l'ensemble de ces jeunes s'accompagne d'une nécessaire mobilité spatiale : que ce soit vers la capitale nationale (Bamako) pour y étudier ; ou vers un pays d'émigration pour y travailler.

\section{Between Individualization and Family Responsibilities: Mobilities of Young Adults Coming from the Region of the Kayes in Mali}

This article examines the case of young adults coming from villages in the region of the Kayes in Mali. They are faced up to the necessity of taking their responsibilities as adults in the family and in the community. However, the Sahelian agriculture is in crisis and do not meet any longer the needs of food or of employment. If their elders overcome this obstacle by migration to France, new generations are now facing another alternative: in their turn migrating, or, studying in order to be more professional in Mali. But this second possibility remains an uncertain option. There is a fine boundary between these two groups: being a potential candidate for emigration or for studies. In any case, the project of social mobility these young adults aspire to, goes along with a necessary spatial mobility, either towards the national capital (Bamako) to study or to any country of migration to work there.

\section{... Entre individualización y responsabilidades familiares: las movilidades de los jóvenes de la región de Kayes en Mali}

Este artículo trata de los jóvenes de los pueblos de la región de Kayes, en Mali, enfrentados a la necesidad de tomar sus responsabilidades de adultos en el seno de su grupo familiar y, más ampliamente, en el seno de su colectividad. Con todo, la crisis de la agricultura en el Sahel ha hecho que ésta haya dejado de ser suficiente para cubrir las necesidades alimentarias y ha dejado de ser un oficio. Si sus mayores pasaron este trance emigrando hacia Francia, las nuevas generaciones se hallan frente a una alternativa, la de emigrar también, o la de estudiar con el fin de profesionalizarse en Mali. Pero esta última eventualidad sigue siendo frágil. La frontera entre ambos grupos, ya sean los candidatos a la emigración o los alumnos y estudiantes, resulta tenue. Sea como fuere, el proyecto de movilidad social al cual aspira el conjunto de estos jóvenes se acompaña de una necesaria movilidad y espacial: ya sea hacia la capital nacional (Bamako) para estudiar allí o hacia un país de emigración para trabajar. 